\section{A better letter}

\section{When space is limited, make every word count, advises Ingrid Eisenstadter.}

T Three years ago, the US-based grantgiving foundation that I work for decided to switch from asking for full grant proposals to asking instead for LOIs, variously called 'letters of inquiry' or 'letters of intent'. These are brief summaries of grant proposals. We made this decision mainly because an LOI is less time-consuming for applicants, an important consideration given that we are a small foundation that must turn away most of the proposals we receive - and also because it is less time-consuming for us.

Many of the LOIs we now receive have similar frailties: some give too much space to introductory discussions and fail to provide enough information about the research protocol. Some use too much technical vocabulary; others neglect to mention how much support they are looking for.

If the foundation does not ask you to specify the sum you are seeking, make sure you have done the research to know that you are within their funding range (and whether they fund internationally, if you live in a different country). If you are way above the giving limit, that is probably sufficient reason for them to turn away your enquiry.

As to technical vocabulary, do not assume that the reviewers will be conversant in the language of dozens of areas of speciality. In our last round of enquiries, we encountered PCL oil-soluble layers, Ancova analysis, fugitive dyes and elastic microbial repertoires, among other topics. Applications that clearly explained the terms began the full evaluation process at the outset, but those that were not clear had to wait for a time when a reviewer could research the terms or search for referees.

A large majority of grant seekers submit their LOIs immediately before the deadline, so you should keep in mind that those submissions will land in a pile-up as the calendar speeds towards the foundation's next board meeting. Thus, if your LOI gets a delayed reading, it risks not getting the detailed attention it deserves.

Most of the grant-makers that ask for LOIs have page-count or word-count limits. Our limit is 1,000 words, but I have seen some as low as 250 words. As you struggle to get your project summary down to that length, take some comfort in knowing that all applicants face the same problem, and sally forth.

LOI instructions do not usually address

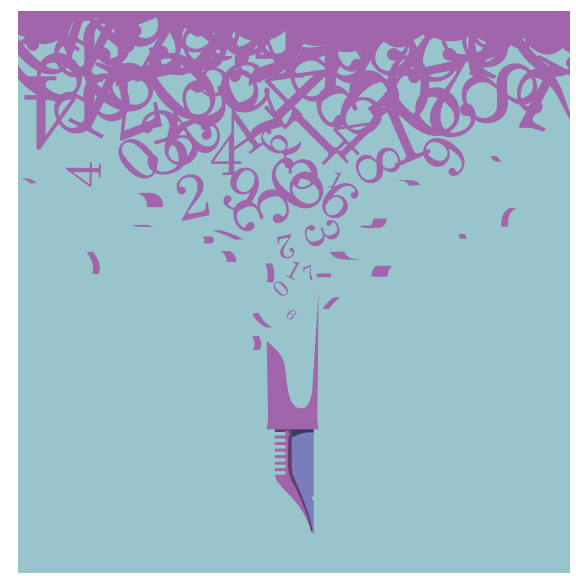

whether illustrations are welcome, although some researchers (not many) do include them. If your LOI would be much clarified by charts, graphs or photos, it is probably worth the gamble to include them. (If you must use an online application form, these uploads will probably not be accommodated.)

E-mail attachments can also pose a problem. When applicants send LOIs as attachments rather than in the body of the e-mail, the documents are often completely anonymous: they do not contain any identifying information. Many of the covering e-mails do not refer to the project title, and if the LOI is filed separately from the e-mail, it can create a serious problem.

So don't do that. Instead of just saying 'LOI attached' in your e-mail, provide a couple of sentences about what makes your proposal attractive or urgent, and include the title of your project and your full identification, including your e-mail address, in the LOI itself as well as in the body of the e-mail.

If you are invited to submit a full grant proposal after your LOI has been reviewed, you have cleared an important hurdle, and your chances of funding are better than when you submit a full grant proposal at the outset of the evaluation process. Leave yourself enough time to pass the final draft of your LOI around to colleagues and get their comments - and don't wait until the evening of the deadline day before you hit 'send'.

Ingrid Eisenstadter is director of grants for the Eppley Foundation for Research in New York.

\section{GRANT AWARDS}

\section{Diversity boost}

A recruitment and retention programme launched by the Howard Hughes Medical Institution (HHMI) in Chevy Chase, Maryland, aims to reduce barriers for women and under-represented minorities who seek academic-research careers in the life sciences. Those barriers include financial hardship, few mentoring and networking opportunities and uncertain career prospects. The Hanna H. Gray Fellows Program will support postdocs for up to four years with a US $\$ 60,000$ annual stipend, plus up to $\$ 20,000$ per year in flexible funds that can be used for family support or other purposes. For eligible fellows who become faculty members, the programme also provides up to $\$ 250,000$ a year, plus $\$ 20,000$ annually in flexible funds for up to four years. That funding will make recipients more attractive to universities, says Barbara Graves, HHMI senior scientific officer. Fellows can tap into HHMI's network of investigators for mentoring and networking, she says. The application deadline is 15 February 2017. See go.nature.com/2d9avh9 for more information.

\section{NETWORKING}

\section{Match.com for mentors}

The US National Institutes of Health (NIH) is rolling out an online tool to match early-career researchers with mentors. The online platform, MyNRMN, asks would-be mentees and mentors to create profiles and connect through direct messages or by joining groups that share their interests. Postdocs, faculty members, staff researchers and administrators can be mentors; mentees can be students and postdocs. The tool is part of the NIHfunded National Research Mentoring Network, which was created in 2014 to improve networking and mentorship opportunities for scientific trainees from diverse backgrounds. More than 2,000 mentees and 1,000 mentors have registered with the programme, says Jamboor K. Vishwanatha, a molecular biologist at the University of North Texas Health Science Center in Fort Worth who is developing the network. Mentees can connect with multiple mentors who share their research interests, location or ethnicity. The platform can also be used to assess the interactions that correlate with later success in scientific careers. "This will allow the development of a 'science' of mentoring and help us to develop best practices," says Vishwanatha. 